\title{
Trapezium-Trapezoid Joint
}

National Cancer Institute

\section{Source}

National Cancer Institute. Trapezium-Trapezoid Joint. NCI Thesaurus. Code C142326.

The articulation of the trapezium and trapezoid bones in the wrist. 